\title{
Use of Imaging During Staging and Surveillance of Localized Colon Cancer in a Large Insured Population
}

\author{
Urshila Durani, MD, MPHa; Dennis Asante, MS; Thorvardur Halfdanarson, MDa; Herbert C. Heien, MSc,d; \\ Lindsey Sangaralingham, MPHc,d; Carrie A. Thompson, MD; Prema Peethambaram, MDa; \\ Fernando J. Quevedo, MDa; and Ronald S. Go, MD ${ }^{\mathrm{c}, \mathrm{e}}$
}

\begin{abstract}
Background: Adherence to surveillance guidelines in resected colon cancer has significant implications for patient morbidity, cost of care, and healthcare utilization. This study measured the underuse and overuse of imaging for staging and surveillance in stage I-II colon cancer. Methods: The OptumLabs database was queried for administrative claims data on adult patients with stage I-II colon cancer who underwent surgery alone in 2008 through 2016. Use of PET and CT imaging was evaluated during both initial staging $(n=6,921)$ and surveillance for patients with at least 1 year of follow-up $(n=5,466)$. "High use" was defined as $>2$ CT abdominal/pelvic (CT A/P) or PET scans per year during surveillance. Results: Overall, $27 \%$ of patients with stage I-II colon cancer did not have a staging CT A/P or PET scan and $95 \%$ did not have a CT chest scan. However, rates of staging CT A/P and CT chest scans increased from $62.0 \%$ (2008) to $74.8 \%$ (2016) and from $2.3 \%(2008)$ to $7.1 \%(2016)$, respectively. Staging PET use was overall very low (5.2\%). During surveillance, approximately $30 \%$ of patients received a CT A/P or PET and $5 \%$ received a CT chest scan within the first year after surgery. Of patients who had surveillance CT A/P or PET scans, the proportion receiving $>2$ scans within the first year (high use) declined from $32.4 \%$ (2008) to $9.6 \%(2016)(P=.01)$. Conclusions: Although PET use remains appropriately low, many patients with stage I-II colon cancer do not receive appropriate staging and surveillance CT chest scans. Among those who do receive these scans during surveillance, high use has declined significantly over time.
\end{abstract}

J Natl Compr Canc Netw 2019;17(11):1355-1361 doi: 10.6004/jnccn.2019.7315

\footnotetext{
${ }^{a}$ Department of Oncology, bepartment of Health Sciences Research, Biomedical Statistics and Informatics, and ${ }^{\mathrm{C} R o b e r t} \mathrm{D}$. and Patricia E. Kern Center for the Science of Health Care Delivery, Mayo Clinic, Rochester, Minnesota; 'OptumLabs, Cambridge, Massachusetts; and 'Division of Hematology, Department of Medicine, Mayo Clinic, Rochester, Minnesota.
}

\section{Background}

Colon cancer is the fourth most common cancer in the United States, and patients are living longer than ever before. ${ }^{1,2}$ Almost $40 \%$ of colon cancers are diagnosed as AJCC stage I or II. ${ }^{2}$ Given the scope of this disease, the implications of surveillance in this population are great. Recommendations for surveillance of stage I-II colon cancer vary by guideline and are summarized in Table 1 . In terms of imaging, NCCN recommends a surveillance CT of the chest and abdomen/pelvis every 6 to 12 months for a total of 5 years for stage II and III disease. $^{3}$ In 2013, ASCO endorsed a set of recommendations from Cancer Care Ontario $(\mathrm{CCO})^{4}$ that include an annual CT of the chest and abdomen for 3 years (stage II and III). ${ }^{4,5}$ Both NCCN and CCO, however, do not recommend routine PET scans either in staging or for surveillance. ${ }^{3,5}$ Other major organizations, including the American Cancer Society and ESMO, have produced similar guidelines. ${ }^{6,7}$

There are several disadvantages to unnecessary imaging, including increased cost of care, radiation exposure, patient anxiety, and incidental findings that can lead to more tests. Given the varying guideline recommendations and recent initiatives to limit unnecessary tests, this study was performed to examine the patterns of imaging use during staging and surveillance in stage I-II colon cancer.

\section{Methods}

\section{Population}

A retrospective analysis of claims data from the OptumLabs Data Warehouse (OLDW) was performed. This database, which includes deidentified claims data for privately insured and Medicare Advantage enrollees in a large, private US health plan, contains claims data on $>100$ million enrollees, representing a diverse mix of

See JNCCN.org for supplemental online content. 
ages, ethnicities, and geographical regions in the United States. The health plan provides comprehensive full insurance coverage for physician, hospital, and prescription drug services. The advantages of OLDW include its wide geographic reach, which provided us with a representative sample of the insured US population and detailed information about patient treatments and procedures. Because this study involved analysis of preexisting, deidentified data, it was exempt from Institutional Review Board approval. ${ }^{8,9}$

Adults aged $\geq 18$ years with a new nonrectal colon cancer diagnosis between January 1, 2008, and December 31,2016 , were included in our staging cohort. To limit our cohort to stage I-II colon cancer with appropriate follow-up, patients were excluded if they did not receive a colectomy (presumed stage IV), received chemotherapy or radiation preoperatively or postoperatively (presumed high-risk stage II or stage III-IV), had a concurrent second cancer, or did not have medical and pharmacy coverage within 90 days before and after colectomy (see supplemental eFigure 1, available with this article at JNCCN.org). Due to the limitations of the OLDW, we were not able to definitively differentiate between stages I and II. However, based on a previous National Cancer Database study and a retrospective institutional study, both of which studied early-stage colon cancer and contained $42 \%$ patients with stage I disease, we estimated that the proportion of patients with stage I cancer in this group would be approximately $40 \% .{ }^{10,11}$ A second surveillance cohort that included patients in the staging cohort with at least 1 year of follow-up after the staging period was created to analyze surveillance strategies.

\section{Definitions}

The staging period was defined as 60 days before to 60 days after surgery. The surveillance period was from 60 days after surgery until death or last follow-up. "High use" in the surveillance cohort was defined as $>2$ CT, PET, or PET/CT scans in 1 year, based on the fact that no current guidelines recommend $>1$ scan every 6 months (2 per year). Supplemental eTable 1 lists the diagnosis, procedure, and imaging codes that were used.

\section{Outcomes}

In the staging cohort, use of PET, PET/CT, CT of the chest, and CT of the abdomen/pelvis (CT A/P) was examined. In the surveillance cohort, the rate of CT, PET, and PET/CT scans was studied. High use was analyzed as a secondary outcome.

\section{Statistical Analysis}

Descriptive statistics were used to define baseline characteristics of the staging and surveillance cohorts and 
to examine the proportion of patients receiving imaging scans during the study period. The CochranArmitage test and chi-square test were used to determine the significance of trends in imaging rates over time. All analyses were performed using SAS 9.3 (SAS Institute Inc).

\section{Results}

A total of 6,921 patients met the inclusion criteria for the staging cohort (supplemental eFigure 1). Baseline characteristics of this group are shown in Table 2, and staging imaging rates are shown in Table 3. Although $72.6 \%$ of patients underwent CT abdomen, CT A/P, or PET imaging during the staging period, only $5.2 \%$ had

\section{Table 2. Characteristics of Staging Cohort}

\begin{tabular}{|c|c|}
\hline & n (\%) \\
\hline Total, N & 6,921 \\
\hline \multicolumn{2}{|l|}{ Age group, y } \\
\hline $18-44$ & 198 (2.9\%) \\
\hline $45-54$ & 767 (11.1\%) \\
\hline $55-64$ & 1,334 (19.3\%) \\
\hline$\geq 65$ & $4,622(66.8 \%)$ \\
\hline \multicolumn{2}{|l|}{ Sex } \\
\hline Female & 3,694 (53.4\%) \\
\hline Male & $3,227(46.6 \%)$ \\
\hline \multicolumn{2}{|c|}{ Race/Ethnicity } \\
\hline Asian & $156(2.3 \%)$ \\
\hline Black & 955 (13.8\%) \\
\hline Hispanic & 403 (5.8\%) \\
\hline White & $5,021(72.5 \%)$ \\
\hline Unknown & $386(5.6 \%)$ \\
\hline \multicolumn{2}{|c|}{ Region of residence } \\
\hline Midwest & $2,238(32.3 \%)$ \\
\hline Northeast & 929 (13.4\%) \\
\hline South & $2,970(42.9 \%)$ \\
\hline West & $766(11.1 \%)$ \\
\hline Unknown & $18(0.2 \%)$ \\
\hline \multicolumn{2}{|c|}{ Year of resection } \\
\hline 2008 & 598 (8.6\%) \\
\hline 2009 & $608(8.8 \%)$ \\
\hline 2010 & 687 (9.9\%) \\
\hline 2011 & $731(10.6 \%)$ \\
\hline 2012 & $898(13.0 \%)$ \\
\hline 2013 & $885(12.8 \%)$ \\
\hline 2014 & $907(13.1 \%)$ \\
\hline 2015 & 747 (10.8\%) \\
\hline 2016 & $860(12.4 \%)$ \\
\hline
\end{tabular}

CT of the chest; $5.8 \%$ of patients underwent PET or PET/CT during the staging period. The proportion of patients receiving CT A/P increased from $62.0 \%$ in 2008 to $74.8 \%$ in 2016 . The rate of PET imaging remained stable over time (5.5\% in 2008 to $6.6 \%$ in 2016; Figure 1).

The surveillance cohort included 5,466 patients from the staging cohort who had at least 1 year of continuous enrollment after the staging period. Approximately $30 \%$ of patients had at least one type of imaging (CT of the abdomen, CT A/P, PET, or PET/CT) during year 1 of followup (Figure 2). Among those with longer follow-up data, this percentage decreased to $18.3 \%$ in year $2,9.3 \%$ in year 3 , $5.1 \%$ in year 4 , and $2.8 \%$ in year 5 . Only $4.6 \%$ of patients underwent CT of the chest during year 1; this percentage decreased to $3.0 \%$ in year $2,1.6 \%$ in year $3,1.0 \%$ in year 4 , and $0.7 \%$ in year 5 (Table 4 ). PET or PET/CT imaging also decreased over time, from $4.0 \%$ in year 1 to $1.7 \%$ in year 2 and $<1 \%$ in years 3 through 5 .

When broken down by year of resection, surveillance CT, PET, and PET/CT imaging during year 1 of follow-up increased from $26.9 \%$ in 2008 to $35.6 \%$ in 2016 (Table 5). Imaging during year 5 of surveillance, however, remained stable, from $18.0 \%$ in 2008 to $18.9 \%$ in 2012. CT chest imaging increased significantly during year 1 of surveillance $(P<.01)$, however, from $3.2 \%$ in 2008 to $8.7 \%$ in 2016 .

High use of surveillance imaging was examined during year 1 of follow-up in the surveillance cohort, and was found to decrease dramatically from $32.4 \%$ in 2008 and $33.8 \%$ in 2009 to $15.9 \%$ in $2010(P<.01)$. This rate decreased even further in 2011 through 2016 (Figure 3). A multivariable logistic regression of age, sex, region of residence, race/ethnicity, and year of resection was performed to determine any sociodemographic predictors of high use; only year of resection predicted high use (Table 6).

\section{Discussion}

Our study demonstrates significant changes in the practice of surveillance and staging of stage I-II colon cancer in the United States over time. The low rate of use of chest CT, despite its recommendation by NCCN,

\section{Table 3. Staging Imaging Rates}

\begin{tabular}{|lc|}
\hline Type of Imaging & Patients, $\mathbf{n}(\%)$ \\
\hline $\begin{array}{l}\text { Any CT of abdomen, CT of abdomen/pelvis, } \\
\text { PET, or PET/CT }\end{array}$ & $5,026(72.6 \%)$ \\
\hline CT of abdomen & $4,887(70.6 \%)$ \\
\hline CT of chest & $357(5.2 \%)$ \\
\hline CT of pelvis & $1,263(18.2 \%)$ \\
\hline PET or PET/CT & $403(5.8 \%)$ \\
\hline
\end{tabular}




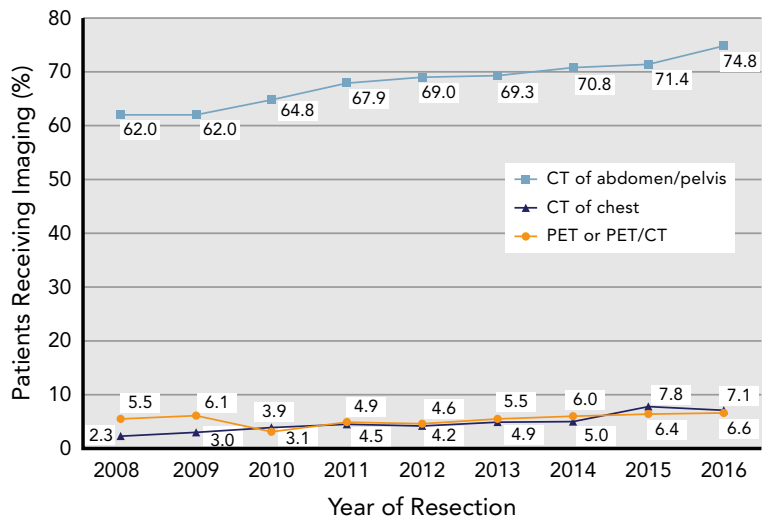

Figure 1. Trends in staging imaging over time.

ASCO, and CCO, is surprising. Although ESMO guidelines do not recommend CT of the chest for staging, they do recommend it for surveillance, ${ }^{6}$ and it is unlikely that oncologists in the United States would consult European guidelines rather than those of NCCN or ASCO. Although a previous study demonstrated close adherence to NCCN treatment recommendations in patients with colon cancer, ${ }^{9}$ our study is the first to demonstrate a low adherence to complete surveillance recommendations. Although it is possible that physicians are not aware of the surveillance recommendations for stage I-II colon cancer, this seems less likely because most patients in this cohort were at least receiving CT A/P during surveillance. Thus, it seems more likely that physicians are choosing not to adhere, for a variety of reasons.

One hypothesis for the markedly low rate of CT chest surveillance is that physicians are using alternative screening methods, such as chest radiography. One retrospective population-based study conducted in Ontario, Canada, showed that between 2002 and 2008 , although only $13 \%$ of the cohort received preoperative CT of the chest/abdomen, $48 \%$ received CT of the abdomen and some form of chest imaging. ${ }^{12}$ Although chest radiography performs poorly compared with chest CT for detection of metastatic disease ${ }^{13}$ there is no evidence that one improves survival compared with the other, and radiography involves less radiation exposure and expense. Thus, because of a lack of strong evidence, physicians may choose to use alternative screening strategies over CT.

Another possible explanation is lack of resources or CT imaging capability. The CCO guidelines do provide a qualifying statement that "[i]f local resources and/or patient preference preclude the use of CT..., a chest X-ray can be substituted for the chest CT. ${ }^{5}$ However, one would presume that if a staging CT of the abdomen was performed (as it was in most of our cohort), then significant barriers to obtaining a CT of the chest are

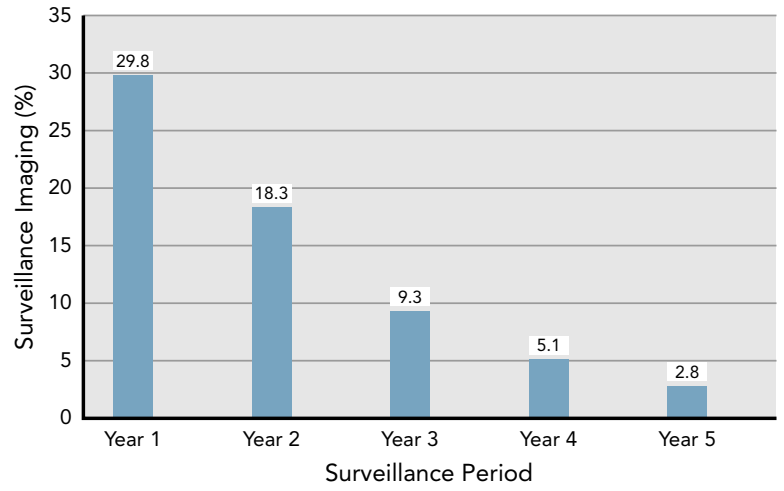

Figure 2. Trends in surveillance imaging according to year after resection.

unlikely. Insurance coverage should not have been a significant barrier, given that most major organizations in the United States, Canada, and Europe recommend CT chest imaging in surveillance of stage II-III colon cancer, and our study group consisted entirely of patients with private insurance or Medicare coverage ${ }^{3,5,7}$ Another possible reason for discrepancies between recommendations and true practice may be the type of treatment facility. Previous work demonstrated that factors such as treatment at NCI-designated facilities predicted more appropriate preoperative staging and treatment in rectal cancer. ${ }^{14}$ Although treatment and staging for this disease differ from nonrectal colon cancer, a similar relationship between facility type, which could not be identified in this study, and surveillance may exist. In the subgroup that did receive surveillance imaging, we hypothesized that high use ( $>2$ scans per year) would be common but would decrease over time, which did occur. The decrease was dramatic from 2009 (33.8\%) to $2010(15.9 \%)$ and then plateaued at approximately $10 \%$ in subsequent years. NCCN Clinical Practice Guidelines in Oncology before 2010 recommended CT imaging yearly for any colorectal cancer at high risk of recurrence (lymphovascular invasion or poorly differentiated tumors). ${ }^{15}$

\begin{tabular}{|lcccc|}
\hline Table & 4. Rates of CT Imaging by Surveillance \\
Period & & & \\
\hline $\begin{array}{l}\text { Surveillance } \\
\text { Perioda }\end{array}$ & $\begin{array}{c}\text { Composite } \\
\text { Imaging }\end{array}$ & $\begin{array}{c}\text { CT of } \\
\text { Chest }\end{array}$ & $\begin{array}{c}\text { CT of } \\
\text { Abdomen/Pelvis }\end{array}$ & $\begin{array}{c}\text { PET or } \\
\text { PET/CT }\end{array}$ \\
\hline Year 1 & $29.8 \%$ & $4.6 \%$ & $28.0 \%$ & $4.0 \%$ \\
\hline Year 2 & $18.3 \%$ & $3.0 \%$ & $17.6 \%$ & $1.7 \%$ \\
\hline Year 3 & $9.3 \%$ & $1.6 \%$ & $9.1 \%$ & $0.5 \%$ \\
\hline Year 4 & $5.1 \%$ & $1.0 \%$ & $5.0 \%$ & $0.3 \%$ \\
\hline Year 5 & $2.8 \%$ & $0.7 \%$ & $2.7 \%$ & $0.2 \%$ \\
\hline
\end{tabular}

aPreviously defined as starting 60 days surgery. 


\begin{tabular}{|c|c|c|c|c|c|c|c|c|c|}
\hline \multicolumn{10}{|l|}{ Composite imaging } \\
\hline Year 1 & $26.9 \%$ & $26.5 \%$ & $26.2 \%$ & $27.7 \%$ & $27.7 \%$ & $29.9 \%$ & $33.5 \%$ & $33.2 \%$ & $35.6 \%$ \\
\hline Year 2 & $27.7 \%$ & $23.8 \%$ & $27.2 \%$ & $27.8 \%$ & $28.0 \%$ & $27.3 \%$ & $31.1 \%$ & $37.6 \%$ & NA \\
\hline Year 3 & $20.3 \%$ & $19.0 \%$ & $22.3 \%$ & $21.0 \%$ & $23.6 \%$ & $24.1 \%$ & $25.7 \%$ & NA & NA \\
\hline Year 5 & $18.0 \%$ & $12.0 \%$ & $20.7 \%$ & $19.0 \%$ & $18.9 \%$ & NA & NA & NA & NA \\
\hline \multicolumn{10}{|l|}{ CT of chest } \\
\hline Year 1 & $3.2 \%$ & $2.0 \%$ & $4.3 \%$ & $5.2 \%$ & $3.7 \%$ & $4.4 \%$ & $4.8 \%$ & $5.9 \%$ & $8.7 \%$ \\
\hline Year 2 & $4.3 \%$ & $4.6 \%$ & $3.9 \%$ & $3.1 \%$ & $5.4 \%$ & $4.4 \%$ & $6.5 \%$ & $5.1 \%$ & NA \\
\hline Year 3 & NA & $4.2 \%$ & $3.3 \%$ & $4.5 \%$ & $4.1 \%$ & NA & $6.3 \%$ & NA & NA \\
\hline Year 1 & $4.8 \%$ & $4.2 \%$ & $5.4 \%$ & $4.1 \%$ & $3.0 \%$ & $3.2 \%$ & $3.8 \%$ & $3.6 \%$ & $4.0 \%$ \\
\hline Year 2 & $3.8 \%$ & NA & $3.4 \%$ & NA & $2.9 \%$ & $2.1 \%$ & $2.4 \%$ & NA & NA \\
\hline Year 3 & NA & NA & NA & NA & NA & NA & NA & NA & NA \\
\hline Year 4 & NA & NA & NA & NA & NA & NA & NA & NA & NA \\
\hline Year 5 & NA & NA & NA & NA & NA & NA & NA & NA & NA \\
\hline
\end{tabular}

Abbreviation: NA, not applicable (cells with $<11$ observations).

In subsequent years, CT imaging surveillance was recommended only for stage II-III colon cancer. ${ }^{3}$ Thus, a move away from surveillance of stage I cancer could be a contributor. However, guidelines are not the only driving factor for whether a test is performed. For example, in addition to patient- and provider-level factors, ${ }^{16}$ changes in billing or health policy/legislation may also contribute to practice patterns and guideline adherence. A meta-analysis in 2007 evaluated 8 randomized controlled trials of intensive follow-up and found an improvement in overall mortality in the intensive group; however, cancer-related mortality was no different between the intervention and control arms, and the studies variably defined both "intensive surveillance" and standard of care follow-up. ${ }^{17}$ When specifically considering trials with CT versus no CT imaging, CT use was not associated with improved survival. ${ }^{17} \mathrm{~A}$ Cochrane review published in 2016 evaluated 15 randomized controlled trials of intensive follow-up strategies for colorectal cancer and did not find any improvement in overall, relapse-free, or colon cancer-specific survival, but it did find a decrease in symptomatic recurrences with intensive follow-up. ${ }^{18}$

Use of PET imaging in colon cancer staging/ surveillance was found to be appropriately low $(6 \%$ in staging, $4 \%$ in year 1 of surveillance). Although the Society of Surgical Oncology's recommendation against PET imaging was not released until 2016, ${ }^{19}$ previous guidelines did not include it as a preferred modality, given the lack of data. A review of the literature in 2011 found that the few studies examining the value of $\mathrm{PET} / \mathrm{CT}$ in preoperative staging were of poor quality, and most were retrospective, demonstrating sensitivities and specificities of $86 \%$ to $100 \%$ and $75 \%$ to $100 \%$, respectively, for detection of synchronous hepatic metastases. ${ }^{20-23}$ In comparison, the sensitivity and specificity of contrast-enhanced CT ranged from $75 \%$ to $98 \%$ and $25 \%$ to $100 \%$, respectively. ${ }^{20-23}$

This finding differs from a previous study showing an increase in PET use, especially within 1 year from surgery

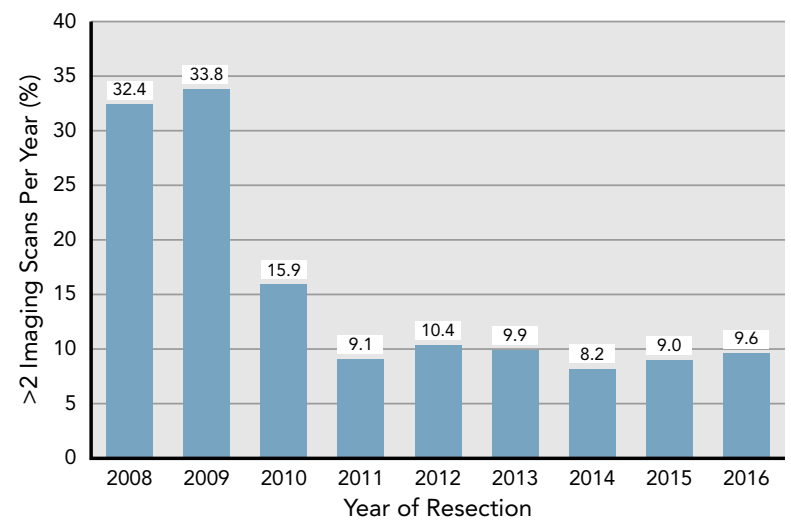

Figure 3. Trends in high use of surveillance imaging over time. 


\begin{tabular}{|c|c|c|}
\hline Variable & $\begin{array}{c}\text { Odds Ratio } \\
(95 \% \mathrm{Cl})\end{array}$ & $P$ Value \\
\hline Age $\geq 65 y$ & $1.21(0.87-1.67)$ & .25 \\
\hline Female sex & $0.97(0.72-1.30)$ & .81 \\
\hline \multicolumn{3}{|c|}{ Region of residence } \\
\hline South & Ref & \\
\hline Midwest & $1.08(0.77-1.51)$ & .66 \\
\hline Northeast & $0.53(0.31-0.92)$ & .02 \\
\hline West & $0.65(0.36-1.16)$ & .14 \\
\hline \multicolumn{3}{|l|}{ Race/Ethnicity } \\
\hline White & Ref & \\
\hline Asian & $0.85(0.32-2.27)$ & .74 \\
\hline Black & $0.85(0.54-1.33)$ & .47 \\
\hline Hispanic & $0.90(0.48-1.70)$ & .75 \\
\hline \multicolumn{3}{|c|}{ Year of resection } \\
\hline 2008 & Ref & \\
\hline 2009 & $0.98(0.59-1.62)$ & .94 \\
\hline 2010 & $0.37(0.21-0.66)$ & $<.001$ \\
\hline 2011 & $0.17(0.09-0.33)$ & $<.001$ \\
\hline 2012 & $0.23(0.13-0.40)$ & $<.001$ \\
\hline 2013 & $0.22(0.12-0.40)$ & $<.001$ \\
\hline 2014 & $0.19(0.11-0.34)$ & $<.001$ \\
\hline 2015 & $0.20(0.11-0.36)$ & $<.001$ \\
\hline 2016 & $0.23(0.12-0.44)$ & $<.001$ \\
\hline
\end{tabular}

(staging/early surveillance period) ${ }^{24}$ Imaging rates in year 1 after surgery increased from $4.8 \%$ in 2001 to $18.8 \%$ in 2008. However, this study examined use specifically in Medicare patients from 2001 to 2008, and included those with stage III cancer and those who received chemotherapy, presumably a higher-risk group. ${ }^{24}$ In addition, PET imaging rates $>1$ year from surgery remained stable $(3.2 \%$ in 2001 to $3.5 \%$ in 2008). Another study examining PET use in 2001 through 2002 found similarly low rates $(8 \%$ in stage II, $3 \%$ in stage I) within the first 2 years of surgery. ${ }^{25}$

Methodologically, our study was limited by the fact that not all of our patients had detailed staging information. Thus, our stage I-II group had to be derived from exclusions based on treatment, and an estimate of the number of patients with stage II disease in our cohort had to be made. In addition, given that the OLDW consists specifically of an insured population, the findings in our study may not apply to patients outside of this group. Lastly, an inherent assumption in this study is that the recommendations from various guideline-establishing groups are evidence-based and result in better care, which is not always the case.
Although a meta-analysis of trials of intensive followup did find an increased rate of salvage surgery in patients undergoing intensive follow-up, presumably because of increased "resectable" recurrences, the definition of intensive follow-up varied with different trials, and overall/cancer-specific survival was not affected. ${ }^{19}$ Results of the PRODIGE 13 trial will show the effect of intensive follow-up (CT imaging alternating with abdominal ultrasound every 3 months) compared with less intensive surveillance (chest radiograph every 6 months with abdominal ultrasound every 3 months) on overall survival. ${ }^{26}$ Despite the fact that all guidelines recommend CT surveillance, the recommended frequency varies. Thus, to provide the most conservative definition of "high use," we set $>2$ scans per year as our threshold because the most frequent surveillance recommendation is for a CT scan twice annually. Even with this definition, a substantial number of patients underwent more than the required number of surveillance scans, which could potentially be a source of anxiety for the patient and may reveal incidental findings that could result in unnecessary diagnostic procedures or more frequent imaging surveillance. Despite these limitations, our study adds new information to the knowledge of survivorship in early-stage colon cancer and suggests the level of adherence to surveillance guidelines.

\section{Conclusions}

Our findings showed appropriately low use of PET imaging and decreasing frequency of CT imaging in surveillance. No predictors of high use other than year of resection were identified. However, chest CT imaging, an important method of surveillance for pulmonary metastases, was not used commonly, despite its recommendation by most guidelines. Further research on why providers are not adhering to certain aspects of surveillance should be performed to gain insight into survivorship decision-making and how guideline adherence can be improved.

Submitted February 19, 2019; accepted for publication April 29, 2019.

Author contributions: Study concept: Durani, Halfdanarson, Thompson, Peethambaram, Quevedo, Go. Funding acquisition: Go. Formal analysis: Asante, Heien. Methodology: Durani, Asante, Heien, Sangaralingham, Go. Supervision: Go. Manuscript preparation: Durani. Review and editing: Asante, Halfdanarson, Heien, Sangaralingham, Thompson, Peethambaram, Quevedo, Go.

Disclosures: The authors have not received any financial consideration from any person or organization to support the preparation, analysis, results, or discussion of this article.

Funding: This study was made possible by a "Transform the Practice" grant from the Mayo Clinic Robert D. and Patricia E. Kern Center for the Science of Health Care Delivery (Go).

Correspondence: Ronald S. Go, MD, Division of Hematology, Department of Medicine, Mayo Clinic, 200 First Street SW, Rochester, MN 55905. Email: go.ronald@mayo.edu 


\section{References}

1. Cancer stat facts: common cancer sites. National Cancer Institute; Surveillance, Epidemiology, and End Results Program website. Available at: https://seer.cancer.gov/statfacts/html/common.html. Accessed August 20, 2019

2. Cancer stat facts: colorectal cancer. National Cancer Institute; Surveillance, Epidemiology, and End Results Program website. Available at: https://seer.cancer.gov/statfacts/html/colorect.html. Accessed August 20, 2019.

3. Benson AB III, Venook AP, Al-Hawary MM, et al. NCCN Clinical Practice Guidelines in Oncology: Colon Cancer. Version 3.2018. To view the most recent version, visit NCCN.org. Accessed October 2, 2018.

4. Meyerhardt JA, Mangu PB, Flynn PJ, et al. Follow-up care, surveillance protocol, and secondary prevention measures for survivors of colorectal cancer: American Society of Clinical Oncology clinical practice guideline endorsement. J Clin Oncol 2013;31:4465-4470.

5. Earle C, Annis R, Sussman J, et al. Follow-up care, surveillance protocol, and secondary prevention measures for survivors of colorectal cancer: guideline recommendations. Available at: https://www. cancercareontario.ca/sites/ccocancercare/files/guidelines/full/ pebc26-2f_0.pdf. Accessed August 20, 2019.

6. Labianca R, Nordlinger B, Beretta GD, et al. Early colon cancer: ESMO clinical practice guidelines for diagnosis, treatment and follow-up. Ann Oncol 2013;24(Suppl 6):vi64-72.

7. El-Shami K, Oeffinger KC, Erb NL, et al. American Cancer Society colorectal cancer survivorship care guidelines. CA Cancer J Clin 2015;65: 427-455.

8. Wallace PJ, Shah ND, Dennen T, et al. Optum Labs: building a novel node in the learning health care system. Health Aff (Millwood) 2014;33: 1187-1194

9. Romanus D, Weiser MR, Skibber JM, et al. Concordance with NCCN Colorectal Cancer Guidelines and ASCO/NCCN Quality Measures: an NCCN institutional analysis. J Natl Compr Canc Netw 2009;7:895-904.

10. Wasif N, Chang YH, Pockaj BA, et al. Association of distance traveled for surgery with short- and long-term cancer outcomes. Ann Surg Oncol 2016;23:3444-3452.

11. Liska D, Stocchi L, Karagkounis G, et al. Incidence, patterns, and predictors of locoregional recurrence in colon cancer. Ann Surg Oncol 2017;24:1093-1099.

12. Mclnnes MD, Nanji S, Mackillop WJ, et al. Utilization of pre-operative imaging for colon cancer: a population-based study. J Surg Oncol 2017; 115:202-207.
13. Peuchot M, Libshitz HI. Pulmonary metastatic disease: radiologic-surgical correlation. Radiology 1987;164:719-722.

14. Charlton ME, Hrabe JE, Wright KB, et al. Hospital characteristics associated with stage II/III rectal cancer guideline concordant care: analysis of Surveillance, Epidemiology and End Results-Medicare Data. J Gastrointest Surg 2016;20:1002-1011.

15. Engstrom PF, Arnoletti JP, Benson AB III, et al. NCCN Clinical Practice Guidelines in Oncology: colon cancer. J Natl Compr Canc Netw 2009;7: 778-831.

16. Ost DE, Niu J, Elting LS, et al. Determinants of practice patterns and quality gaps in lung cancer staging and diagnosis. Chest 2014;145: 1097-1113.

17. Tjandra JJ, Chan MK. Follow-up after curative resection of colorectal cancer: a meta-analysis. Dis Colon Rectum 2007;50:1783-1799.

18. Jeffery M, Hickey BE, Hider PN, et al. Follow-up strategies for patients treated for non-metastatic colorectal cancer. Cochrane Database Syst Rev 2016;11:CD002200

19. Zinzani PL, Stefoni $\mathrm{V}$, Tani M, et al. Role of $\left[{ }^{18} \mathrm{~F}\right]$ fluorodeoxyglucose positron emission tomography scan in the follow-up of lymphoma. J Clin Oncol 2009;27:1781-1787.

20. Brush J, Boyd K, Chappell F, et al. The value of FDG positron emission tomography/computerised tomography (PET/CT) in pre-operative staging of colorectal cancer: a systematic review and economic evaluation. Health Technol Assess 2011;15:1-192, iii-iv.

21. D'Souza MM, Sharma R, Mondal A, et al. Prospective evaluation of CECT and ${ }^{18} \mathrm{~F}-\mathrm{FDG}-\mathrm{PET} / \mathrm{CT}$ in detection of hepatic metastases. Nucl Med Commun 2009;30:117-125.

22. Kong G, Jackson C, Koh DM, et al. The use of 18F-FDG PET/CT in colorectal liver metastases-comparison with CT and liver MRI Eur J Nucl Med Mol Imaging 2008;35:1323-1329.

23. Ramos E, Martínez L, Gámez C, et al. Use of PET-CT in pre-surgical staging of colorectal cancer hepatic metastases [in Spanish]. Cir Esp 2008;84 71-77.

24. Bailey CE, Hu CY, You YN, et al. Variation in positron emission tomography use after colon cancer resection. J Oncol Pract 2015;11:e363-372.

25. Zafar HM, Mahmoud NN, Mitra N, et al. Resected colorectal cancer among Medicare beneficiaries: adoption of FDG PET. Radiology 2010; 254:501-508

26. Lepage C, Phelip JM, Cany L, et al. Effect of 5 years of imaging and CEA follow-up to detect recurrence of colorectal cancer: the FFCD PRODIGE 13 randomised phase III trial. Dig Liver Dis 2015;47:529-531. 
Supplemental online content for:

\section{Use of Imaging During Staging and Surveillance of Localized Colon Cancer in a Large Insured Population}

Urshila Durani, MD, MPH; Dennis Asante, MS; Thorvardur Halfdanarson, MD; Herbert C. Heien, MS; Lindsey Sangaralingham, MPH; Carrie A. Thompson, MD; Prema Peethambaram, MD;

Fernando J. Quevedo, MD; and Ronald S. Go, MD

J Natl Compr Canc Netw 2019;17(11):1355-1361

eFigure 1: CONSORT Diagram of the Study Cohort

eTable 1: Diagnosis and Procedure Codes 


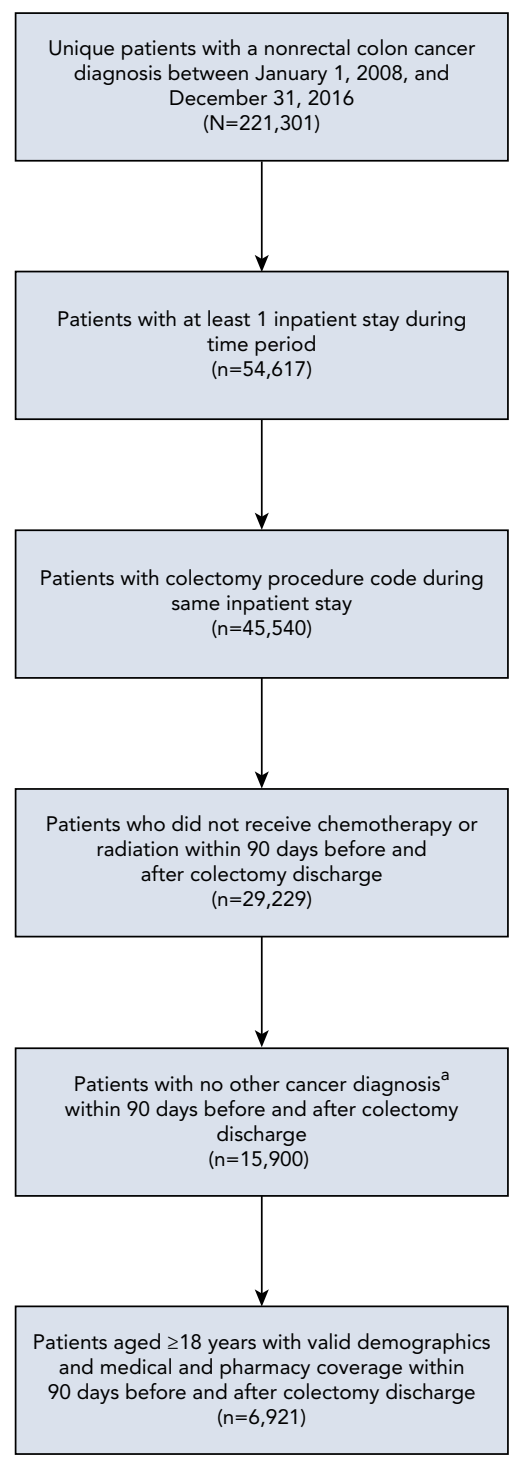

\section{eTable 1. Diagnosis and Procedure Codes}

Diagnosis/Procedure Codes

Nonrectal colon cancer ICD-9-CM: 153.x

ICD-10-CM: C18.x

Colectomy CPT: 44140-44160, 44204-44212

\begin{tabular}{|ll|} 
& ICD-9-CM: 17.31-17.39, 45.71-45.75, 45.76, \\
& 45.79, 45.81-45.83, 48.61, 48.69 \\
& ICD-10-CM: ODTEx, ODTFx, ODTGx, ODTHx, \\
& ODTKx, ODTLx, ODTMx, ODTNx \\
\hline CT of abdomen & CPT: $74150-74178$ \\
\hline CT of chest & CPT: $71250-71270$ \\
\hline CT of pelvis & CPT: $72102-72104$ \\
\hline PET or PET/CT & CPT: $78811-78816$ \\
\hline
\end{tabular}

eFigure 1. CONSORT diagram of the study cohort. ${ }^{a}$ Nonmelanoma skin cancers were allowed. 